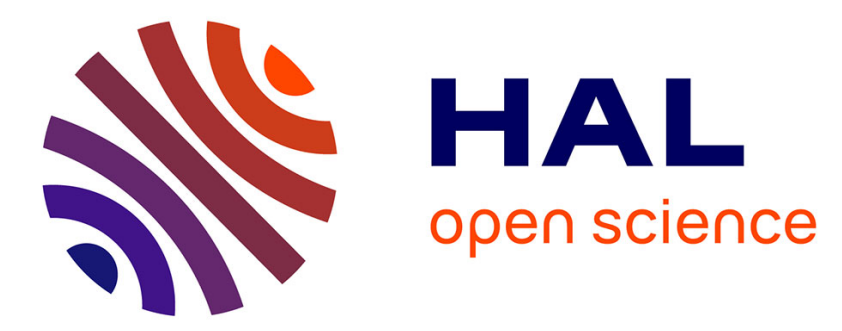

\title{
La démarche supply chain management vue par les salariés d'une multinationale de la haute technologie
}

Joëlle Morana

\section{To cite this version:}

Joëlle Morana. La démarche supply chain management vue par les salariés d'une multinationale de la haute technologie. Logistique \& Management, 2007, 15 (2), pp. 81-92. halshs-00284787

\section{HAL Id: halshs-00284787 https://shs.hal.science/halshs-00284787}

Submitted on 3 Jun 2009

HAL is a multi-disciplinary open access archive for the deposit and dissemination of scientific research documents, whether they are published or not. The documents may come from teaching and research institutions in France or abroad, or from public or private research centers.
L'archive ouverte pluridisciplinaire HAL, est destinée au dépôt et à la diffusion de documents scientifiques de niveau recherche, publiés ou non, émanant des établissements d'enseignement et de recherche français ou étrangers, des laboratoires publics ou privés. 


\section{La démarche Supply Chain Management vue par les salariés d'une multinationale de la haute technologie}

\author{
Joëlle MORANA \\ Maître de Conférences, Université de Lyon II, LET, CNRS \\ jmorana@yahoo.fr
}

Déterminer et évaluer les axes de progrès du Supply Chain Management (SCM) est primordial. Cet article s'intéresse à cette problématique par une étude effectuée auprès d'employés d'une multinationale du secteur de la haute technologie. Pour cela, un questionnaire est proposé, qui décrit neuf thèmes qui définissent les facteurs clés de succès du SCM. Les résultats - par une analyse univariée et une analyse en composantes principales - permettent de classifier les thèmes et les items jugés importants par les répondants. Quatre axes de progrès sont considérés : un axe stratégique, un axe socio-organisationnel, un axe technologique et un axe 'capacité de réponse'. Les réponses obtenues permettent également de situer la perception de la pratique de ce type de démarche, et d'ajuster - si besoin - des actions correctives pour une diffusion plus fine.

\section{Introduction}

Le présent article part du constat selon lequel le Supply Chain Management (SCM) - en tant que démarche stratégique de gestion - fait l'objet de questionnements de plus en plus poussés sur sa mesure. Idéalement, pour assurer la réussite de tout projet (tel que peut-être considéré le SCM), il convient d'énoncer clairement la problématique et de réfléchir sur les impacts technologiques, humains et organisationnels dudit projet. Autrement dit, pour chacun de ces éléments, l'identification des différents besoins, suivi de leur maîtrise doit en assurer sa mise en œuvre, sa mise en place, sa pérennisation. Or, dans le cadre du SCM, une des premières difficultés à sa mesure est l'absence d'une définition normalisée ${ }^{1}$. Ce faisant, la littérature maintenant abondante sur le thème du SCM permet d'entériner un certain nombre de dimensions, elles-mêmes décomposées en items aptes à être utilisés comme mode opératoire. L'objet auquel l'article tente d'apporter des éléments de réponse est : peut-on concevoir un questionnaire apte à faciliter la mesure du SCM, à travers l'énumération de dimensions et items clés ?

La grille de lecture qui est proposée s'appuie sur une analyse de travaux de la Michigan State University, de l'Ohio State University et d'un regroupement d'auteurs mené par John T. Mentzer. L'optique est de repérer les idées phares mises en exergue et les composants s'y référant. L'ensemble montre des réflexions approfondies mais pour autant peu de tests in vivo. De là, le choix fait par l'auteur de s'appuyer sur une étude menée auprès d'entreprises portant sur le Total Quality Management dans une perspective de chaîne d'approvi-
1 - Parmi les dernières définitions proposées, il est possible de retenir la définition $d u$ Council of SCM Professionals qui précise que le «SCM comprend la planification et la gestion de toutes les activités relevant de la recherche de fournisseurs, de l'approvisionnement, de la transformation et toutes les activités relevant du management logistique. Cela inclut tout particulièrement la coordination et la collaboration entre les partenaires du canal, qui peuvent être des fournisseurs, des intermédiaires, des prestataires de services et des clients. En soi, le SCM intègre donc le management de l'offre et de la demande, au sein et entre les entreprises » (Gibson et al., 2005). 
sionnement (Forker et al., 1997) : questionnaire jugé finalisé et utilisable dans le cadre de l'étude ici menée - sous couvert, il va de soi, d'amendements pour rejoindre une philosophie SCM.

\section{Vers une détermination des dimensions et items clés du SCM}

Plusieurs écoles et plusieurs auteurs ont défini les processus ${ }^{2}$ du SCM. Dans le cadre de cet article, nous poserons un regard sur trois travaux jugés représentatifs des réflexions actuelles sur le SCM : ceux de la Michigan State University qui présente la 'supply chain structure 2000' (GRLT, 1995 ; Bowersox et al., 1999), ceux de l'Ohio State University (Cooper et al., 1997 ; Lambert et al., 1998) et ceux du groupe de travail de Mentzer et al. (2001) suivis par l'étude de Min et Mentzer (2004).

Dans la perspective de la Michigan State University (GRLT, 1995 ; Bowersox et al., 1999), le SCM associe trois "contextes". En premier lieu, le contexte 'opérationnel' recommande l'intégration des parties prenantes dans un projet conjoint. A travers ce contexte, l'objectif recherché est de maximiser les efforts de standardisation entre acteurs amont, interne et aval de la chaîne d'approvisionnement. En deuxième lieu, le contexte 'planification et contrôle' recherche la compatibilité des technologies de l'information et de la communication. Son orientation est - via une technologie optimale, une planification et un contrôle - de permettre aux différentes parties prenantes d'avoir connaissance de l'état des systèmes et de mettre en cohérence les différentes ressources en présence. Enfin, en troisième lieu, le contexte 'comportemental' souligne la coordination des acteurs avec une définition des rôles de chacun et un partage des profits et risques. De l'analyse de ces travaux, il est possible de retenir deux préconisations centrales, à savoir (1) l'importance de l'étude, modélisation et évaluation des connexions au sein des processus ceci afin d'assurer une réelle efficacité du SCM et (2) la recherche d'une même finalité, d'une même standardisation dans la conception d'un produit et d'un service.

Dans les travaux de l'Ohio State University (Cooper et al., 1997 ; Lambert et al., 1998), trois éléments premiers composent le SCM : la structure relationnelle du réseau de la chaîne d'approvisionnement, les processus managériaux de la chaîne d'approvisionnement et la gestion des composants. L'objet principal de la structure relationnelle du réseau de la chaîne d'approvisionnement est d'identifier le nombre et le positionnement critique des acteurs dans la chaîne. Pour les processus managériaux, l'optique est ici de désigner et d'analyser les processus de chaque activité. Divisé en une composante physique et technique et une composante managériale et comportementale, le troisième élément considère que "si les composantes managériales et comportementales ne sont pas alignés pour conduire et renforcer un comportement organisationnel en support des objectifs et des opérations de la supply chain, la supply chain sera probablement moins compétitive » (Lambert et al., 1998). En regard de ces différents éléments, deux points importants apparaissent, à savoir que le SCM (1) s'apprécie au-delà d'une chaîne d'approvisionnement 'classique' : fournisseur-entreprise-distributeur-client mais au contraire ramifie plusieurs fournisseurs, plusieurs entreprises et plusieurs distributeurs en vue de délivrer un bien et un service à un client final et (2) se présente comme une philosophie qui touche la culture même de chaque organisation.

Mentzer et al. (2001) proposent deux construits afin de structurer le SCM : une "Supply Chain Orientation" ("la mise en place par une organisation d'implications systémiques et stratégiques concernant les activités tactiques impliquées dans la gestion des différents flux de la chaîne d'approvisionnement") et un "Supply Chain Management" ("la coordination systémique, stratégique et la gestion tactique des actions au sein des départements d'une organisation particulière, ainsi que des affaires menées à l'intérieur de la chaîne d'approvisionnement. Il a pour but d'améliorer la performance long terme de chaque organisation et de la chaîne d'approvisionnement des organisations dans leur ensemble”) (p. 18). S'appuyant sur cette configuration, Min et Mentzer développent en 2004 un questionnaire en vue de mesurer ces deux construits. Cependant, une troisième échelle de mesure $[\mathrm{PERF}]$ relative à la performance de la firme est développée dans le but de tester la validité nomologique des deux échelles SCO et SCM. Pour récapituler, l'objet de ce travail est de proposer une liste d'items apte à évaluer le SCM dans les différentes dimensions qui le composeraient. Ainsi, la première échelle SCO comprend 6 facteurs et un total de 20 items, la deuxième échelle SCM comprend 7 facteurs et un total de 29 items et enfin, la troisième échelle PERF comprend 5 facteurs et 
Tableau 1 : Structuration de la littérature sur le SCM

\begin{tabular}{|c|c|c|}
\hline GRLT (1995) $\Rightarrow$ Bowersox et al. (1999) & Cooper et al. (1997) - Lambert et al. (1998) & Mentzer et al. (2001) $\Rightarrow$ Min et Mentzer (2004) \\
\hline $\begin{array}{l}\text { Contexte opérationnel } \\
\text { 1. Intégration du client } \\
\text { - Segmentation des clients } \\
\text { - Pertinence de chaque demande } \\
\text { - Réponse à chaque demande } \\
\text { - Flexibilité aux circonstances non planifiées } \\
\text { 2. Intégration interne } \\
\text { - Union des fonctions départementales } \\
\text { - Procédures standards, simples, conformes } \\
\text { - Adaptation structurelle } \\
\text { 3. Intégration du fournisseur de matières } \\
\text { et de services } \\
\text { - Sur un plan stratégique, financier, opérationnel } \\
\text { et de management }\end{array}$ & $\begin{array}{l}\text {....> physiques et techniques (planning et méthodes } \\
\text { de contrôle, rythmes de travail/structure de } \\
\text { l'activité, structure de l'organisation, } \\
\text { communication et flux d'information de la } \\
\text { structure technique, flux du produit de la structure } \\
\text { technique) } \\
\text {...> managériales et comportementales (méthodes de } \\
\text { management, structure de pouvoir et de } \\
\text { leadership, structure des risques et } \\
\text { récompenses, culture et attitude) }\end{array}$ & $\begin{array}{l}\text { Performance (PERF) } \\
\ldots .>\text { Disponibilité : stocks, stocks de sécurité } \\
\ldots .>\text { Offre des produits et services : variété, qualité par } \\
\quad \text { rapport aux concurrents } \\
\ldots .>\text { Caractère d'actualité : livraison et information } \\
\quad \text { en JAT } \\
\ldots .>\text { Profitabilité : retour financier } \\
\ldots .>\text { Croissance : CA, parts de marché }\end{array}$ \\
\hline $\begin{array}{l}\text { Contexte de planning et de contrôle } \\
\text { 1. Technologie et Planification } \\
\text { - Gestion de l'information et de la communication } \\
\text { interne } \\
\text { - Connexion entre acteurs } \\
\text { - Collaboration prévisionnel/planning } \\
\text { 2. Mesure } \\
\text { - Evaluation fonctionnelle } \\
\text { - Méthodologies d'évaluation } \\
\text { - Métriques } \\
\text { - Impact financier }\end{array}$ & $\begin{array}{l}\text { - Processus managériaux de la supply chain } \\
\ldots \text {...> Accroissement de l'efficacité et diminution } \\
\text { des frictions } \\
\text {...> identification et analyse des processus de chaque } \\
\quad \text { activité }\end{array}$ & $\begin{array}{l}\text {... Supply chain orientation (SCO) } \\
\text {..> Crédibilité : notions de fiabilité, reconnaissance de } \\
\text { l'entreprise, pas de fausse réclamations ; } \\
\ldots .>\text { Bienveillance : 'santé' de l'entreprise, réponses } \\
\text { aux questions, partage des risques et } \\
\text { récompenses; } \\
\ldots .>\text { Engagement : coopération, aide technique ; } \\
\ldots .>\text { Top Management Support : diffusion des } \\
\text { objectifs, contrats à long terme, formation } \\
\ldots .>\text { Compatibilité : stratégie et culture communes } \\
\ldots .>\text { Normes : coopération, création de valeur }\end{array}$ \\
\hline $\begin{array}{l}\text { Contexte comportemental } \\
\text { 1. Intégration relationnelle } \\
\text { - Clarté des rôles de chaque intervenant } \\
\text { - Lignes de conduite communes } \\
\text { - Partage de l'information } \\
\text { - Partage des gains et risques }\end{array}$ & $\begin{array}{l}\Rightarrow \text { Structure relationnelle } \\
\ldots .>\text { Nombre de tiers le long de la chaîne } \\
\ldots .>\text { Nombre de fournisseurs et de clients à l'intérieur } \\
\text { de chacun des tiers } \\
\ldots .>\text { Identification des membres de } 1^{\text {er }} \text { ordre et de } \\
\quad \text { support } \\
\ldots .>\text { Positionnement de la société sur la supply chain }\end{array}$ & 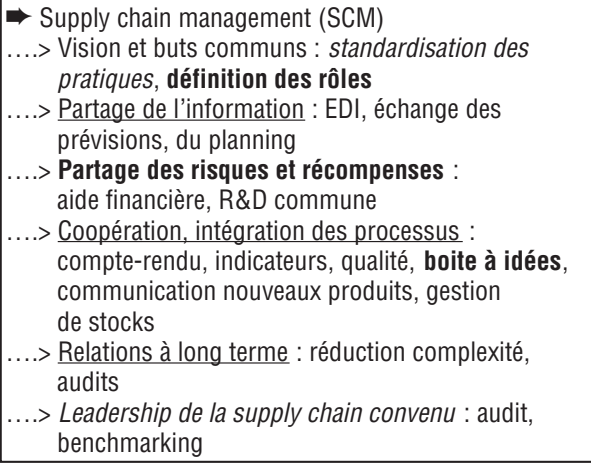 \\
\hline
\end{tabular}

Légende : les mots en gras sont associés à I'humain, les mots soulignés à la technologie, les mots en italique à I'organisationnel

15 items. L'idée principale à retenir ici, selon nous, est la volonté manifeste à la détermination d'une 'mesure pragmatique' du SCM. Ce faisant, comme nous le verrons dans le paragraphe suivant et bien que ce travail académique soit conséquent, nous avons fait le choix d'utilisation d'un autre questionnaire : questionnaire jugé plus « intéressant » car testé empiriquement auprès d'entreprises.

Le tableau 1 tente de rapprocher ces trois réflexions. Cette mise à plat constitue une première grille d'analyse dont l'optique est de dégager des lignes de conduites communes.

A l'issue de cette structuration, nous proposons «d'extraire » les mots clés que nous rapprocherons - autant que faire se peut - à l'objet et mots clés du questionnaire utilisé de Forker et al. (1997). Le découpage s'établit tel que suit : s'appuyant sur l'idée d'un découpage en impacts technologiques, humains et organisationnels d'un projet, le tableau 2 présente une liste de codifications par mots/idées clés tels qu'apparus dans le tableau 1 .

\section{Vers une mise en application au sein d'une multinationale : le construit du questionnaire utilisé}

Au sein de l'organisation étudiée, dénommée Alpha pour des raisons de confidentialité, la démarche SCM a consisté à l'origine en une réorganisation des schémas logistiques au sein des unités d'assemblage, conduisant à la refonte des systèmes informatiques par la mise en place de progiciels ERP et de technologies MRP. Désormais, la démarche s'étend en amont aux usines fabricant les produits et en aval vers les clients du groupe qui ont en ligne une totale visibilité des commandes passées (traçabilité des mises en production puis 
Tableau 2 : Essai de rapprochement entre impacts technologiques, humains et organisationnels et la "structuration de la littérature sur le SCM : Tableau 1 »

\begin{tabular}{|c|c|c|c|}
\hline Impacts & Mots & Auteurs & code \\
\hline Technologiques & $\begin{array}{l}\text { - Contexte de planning et de contrôle } \\
\text { - Performance } \\
\text { - Partage de l'information } \\
\text { - Coopération, intégration des processus } \\
\end{array}$ & $\begin{array}{l}\text { Bowersox et al. (1999), Lambert et al. (1998) } \\
\text { Min et Mentzer (2004) } \\
\text { Min et Mentzer (2004) } \\
\text { Min et Mentzer (2004) } \\
\end{array}$ & $\begin{array}{l}\text { T1 } \\
\text { T2 } \\
\text { T3 } \\
\text { T4 }\end{array}$ \\
\hline Humains & $\begin{array}{l}\text { - Clients, Fournisseurs } \\
\text { - Départements } \\
\text { - Contexte comportemental dans son ensemble, à rapprocher } \\
\text { aux composantes managériales et comportementales + culture } \\
\text { commune/ définition des rôles/partage des risques et récompenses } \\
\text { - Rythmes de travail } \\
\text { - Diminution des frictions } \\
\text { - Nombre de tiers } \\
\text { - Top management } \\
\text { - Formation } \\
\text { - Boite à idées }\end{array}$ & $\begin{array}{l}\text { Bowersox et al. (1999), Lambert et al. (1998) } \\
\text { Bowersox et al. (1999) } \\
\text { Bowersox et al. (1999), Lambert et al. (1998), Min et Mentzer } \\
\text { (2004) } \\
\text { Lambert et al. (1998) } \\
\text { Lambert et al. (1998) } \\
\text { Lambert et al. (1998) } \\
\text { Min et Mentzer (2004) } \\
\text { Min et Mentzer (2004) } \\
\text { Min et Mentzer (2004) }\end{array}$ & $\begin{array}{c}\mathrm{H} 4 \\
\mathrm{H} 5 \\
\mathrm{H} 6 \\
\mathrm{H} \\
\mathrm{H} 8 \\
\mathrm{H} 9\end{array}$ \\
\hline Organisationnels & $\begin{array}{l}\text { - Standardisation des procédures/pratiques } \\
\text { - Adaptation structurelle/analyse des processus/positionnement de la Ste } \\
\text { - SCO dans sa grande majorité } \\
\text { - SCM dans sa grande majorité }\end{array}$ & $\begin{array}{l}\text { Bowersox et al. (1999), Min et M. (04) } \\
\text { Bowersox et al. (1999), Lambert et al. ('98) } \\
\text { Min et Mentzer (2004) } \\
\text { Min et Mentzer (2004) }\end{array}$ & $\begin{array}{l}01 \\
02 \\
03 \\
04\end{array}$ \\
\hline
\end{tabular}

des acheminements). Ajoutons qu' Alpha propose diverses formations internes en matière de SCM.

Dans le cadre du construit du questionnaire utilisé, l'approche que nous avons retenue consiste à « superposer » une étude de terrain (quantitative) portant sur la performance du Total Quality Management (TQM) dans la perspective d'une supply chain et les éléments clés ressortant du tableau 2. Selon nous, le rapprochement TQM/SCM peut être probant en ce sens que le lien qualité/logistique est l'objet de plusieurs publications dans la littérature académique. Ainsi, très tôt, la relation entre ces deux champs de gestion fut montrée (Wagoner, 1989). De même entre le TQM et le SCM (Forker et al., 1997), soulignant pour ces deux approches leur volonté à "aller main dans la main” (Flynn et Flynn, 2005, p. 3434).

Dans le cadre de notre étude, le choix a donc été de s'appuyer sur le questionnaire développé par Forker et al. (1997). Ce choix fut fait sur l'idée selon laquelle le test réel du questionnaire auprès d'entreprises augurait d'une validité empirique pouvant servir de socle à notre propre analyse portant sur le SCM. Le tableau 3 met ainsi en exergue les liens entre les huit dimensions décrites pour mesurer la performance du TQM, les points clés propres au SCM tels qu'énoncés dans le paragraphe précédent (Cf. Tableau 2) et le 'construit' final du questionnaire SCM utilisé.

En l'état, l'étude de Forker et al. apparaît comme particulièrement intéressante car chaque thème fait l'objet d'une liste d'items adaptable à une mesure du SCM. Nous pou- vons ainsi retrouver presque tous les points soulignés dans le tableau 2. Peut-être cependant convient-il de mieux mettre en exergue la relation avec le client final et celle avec les autres parties prenantes (tiers) qui semblent manquer. Aussi, un certain nombre de modifications a été fait sur les thèmes et items proposés, tels que :

- La substitution du concept de qualité pour le concept de supply chain ou SCM,

- L'introduction à une référence inter-organisationnel lorsque celle-ci s'avérait nécessaire, ceci afin de souligner le caractère systémique et étendu du SCM,

- La séparation du thème "la gestion de la qualité du fournisseur" en deux thèmes, se référant respectivement à (1) l'implication des parties prenantes au SCM (\# clients finaux non inclus) et (2) les clients finaux et le SCM,

- Le remplacement de certains termes anglo-saxons considérés comme trop littéraires après un pré-test qualitatif du questionnaire (les répondants sont de différentes nationalités).

\section{Synthèse des principaux résultats : Une structure en quatre axes de progrès du SCM}

Le tri à plats ainsi que l'analyse factorielle expriment les souhaits des répondants dans le rôle attendu au SCM. Le tableau 4 souligne les éléments de synthèse issus des calculs effectués dont le détail se trouve dans les Annexes A et B. 
Tableau 3 : Questionnaire de Forker et al. (1997) - liens avec le tableau 2 et construit du questionnaire final

1. Thèmes du questionnaire de Forker et al. (1997)

2. Relation Qualité (Cf. Codes Tableau 2) / SCM

3. Thèmes finaux du questionnaire SCM

1. Thème 1 Forker et al. (1997) : Le rôle du Top Management dans la politique de qualité (13 items) Objet : Mise en place d'une politique de qualité au plus haut niveau de la structure organisationnelle. Mots-clés : adoption, long terme, coûts, calendriers, réunions de suivi, plan.

2. Lien(s) avec le SCM (Tab. 2) : H7, 03, 04, T1

3. Thème 1 questionnaire SCM : Le rôle du Top Management et de la politique SCM (13 items)

1. Thème 2 Forker et al. (1997) : Le design du produit et services (6 items)

Objet: Recherche d'une qualité constante tout au long de la chaîne intra organisationnelle.

Mots-clés : contrôle, coordination, spécifications, procédures, mise en œuvre, productivité, ser vices internes.

2. Lien(s) avec le SCM (Tab. 2) : T1, T2, T3, T4, H2

3. Thème 2 questionnaire SCM : Le design du produit et services (6 items)

1. Thème 3 Forker et al. (1997) : La gestion de la qualité du fournisseur (8 items)

Objet: Recherche d'une qualité suffisante dès le départ du cycle de réalisation du produit et du ser vice.

Mots-clés : sélection, qualification, formation, assistance technique, implication, long terme, clarté des spécifications.

2. Lien(s) avec le SCM (Tab. 2) : H1, 03, $04 \Rightarrow$ Rappel : Thème séparé en 2

3. Thème 3 quest. SCM : Implication des parties prenantes dans Ie SCM (hors clients finaux) (8 items)

4. Thème 4 questionnaire SCM : Le SCM et les clients finaux ( 5 items)

1. Thème 4 Forker et al. (1997) : Le rôle du département qualité (5 items)

Objet: Reconnaissance du département dédié à la qualité.

Mots-clés: visibilité, reconnaissance, prise de décision, coordination intra organisationnelle

2. Lien(s) avec le SCM (Tab. 2) : H2, H7

3. Thème 5 quest. SCM : Rôle de la cellule qui est en charge avec le déploiement du SCM (5 items)

1. Thème 5 Forker et al. (1997) : Les procédures de gestion de process et opérationnelles (6 items)

Objet: Correcte appréhension et sans retour sur les processus de biens et ser vices.

Mots-clés: visibilité, reconnaissance, prise de décision, coordination intra organisationnelle

2. Lien(s) avec le SCM (Tab. 2) : 01, 02, 03, 04

3. Thème 6 questionnaire SCM : Management des procédures - processus (6 items)

1. Thème 6 Forker et al. (1997) : La qualité des données et des reportings ( 8 items) Objet : Pertinence et visibilité des métriques. Mots-clés : visualisation, indicateurs, pertinence, communication/information

2. Lien(s) avec le SCM (Tab. 2) : T1, T2, T3, T4

3. Thème 7 questionnaire SCM : Qualité des données et des reportings (16 items : Nb. : ajout d'items sur la définition de la forme des reportings)

1. Thème 7 Forker et al. (1997) : Les relations avec les employés et la formation (8 items)

Objet : Reconnaissance du travail fourni par les salariés.

Mots-clés: réciprocité des échanges, implication, responsabilisation

2. Lien(s) avec le SCM (Tab. 2) : H1 à H9, $\mathrm{O3}$

3. Thème 8 questionnaire SCM : Relations avec les employés (8 items)

1. Thème 8 Forker et al. (1997) : La formation (8 items)

Objet: Explication aux employés des enjeux de la qualité et aide à l'utilisation d'outils statistiques.

Mots-clés: Moyens et information

2. Lien(s) avec le SCM (Tab. 2) : T1, 03, 04

3. Thème 9 questionnaire SCM : Formation (8 items)

De ces résultats, nous suggérons d'apprécier le SCM selon quatre axes de progrès, comprenant deux thèmes centraux chacun.

\section{1. l'Axe Stratégique : La mise en place et le suivi}

Au-delà de l'idée selon laquelle il convient de pratiquer le SCM, la question de son appropriation est centrale. Le SCM requiert un changement profond de l'organisation et des organisations impliquées dans un réseau d'affaires. Un réseau entre fonctions et entreprises est subordonné à l'intention du Top Management de mettre en place les moyens humains, financiers et structurels pour conclure ce projet. L'adoption du SCM par chacun peut être aidée par la constitution d'une cellule dédiée pour son suivi et son appréciation. Ceci prend part à la durabilité d'un tel projet dont il n'est pas toujours aisé de dessiner l' architecture. Ce premier point trouve toute sa justification dans les idées clés telles que soulignées dans la rubrique «impacts organisationnels » du tableau 2. 


\section{Méthodologie de recherche}

Quoique le questionnement se fonde sur une analyse des dimensions du Supply Chain Management qui en l'état présuppose I'interaction de plusieurs parties prenantes d'une chaîne d'approvisionnement, la présente étude se focalise sur la présentation d'une seule entreprise : I'entreprise Alpha. Les raisons de ce choix sont multiples. Alpha est une multinationale du secteur de la haute technologie. Dans ce secteur d'activité, I'analyse de sa politique SCM est intéressante compte-tenu des contraintes subies telles qu'une forte concurrence et une durée de vie du produit relativement courte (en moyenne 2 ans). En outre, l'intérêt de cette firme pour une mise en ouvre d'une approche SCM est relativement ancienne (plus de 10 ans) et conforte également le choix d'une analyse de ladite firme. De même, à noter une place " pivot » de la structure étudiée dans sa chaîne d'approvisionnement. Un bémol cependant doit dès à présent être souligné : l'interrogation de la seule firme (et de ses filiales). Nous aurions aimé questionner d'autres acteurs de cette supply chain mais ce secteur relativement confidentiel a rendu cette tâche non réalisable.

La collecte des données a été réalisée par l'envoi de questionnaires auto-administrés (en langue anglaise) auprès des différentes filiales françaises et étrangères d'Alpha, soit un envoi sur 14 pays. Compte tenu du caractère géographiquement très éclaté des filiales d'Alpha, le choix a été de transmettre ce dernier par voie électronique, ce qui permet un envoi sur des distances importantes à un coût très faible. Un total de 515 questionnaires a été envoyé.

Après deux relances, 82 personnes ont répondu. La répartition par continent/pays s'établit telle que suit : 1 répondant d'Afrique (Maroc) ; 17 d'Asie (1 Chine, 3 Japon, 1 Corée, 7 Malaisie, 5 Singapour) ; 60 d'Europe (2 Allemagne, 2 Finlande, 22 France, 27 Italie, 1 Pays Bas, 1 Suède) ; 4 Amérique du Nord (Etats-Unis). Différents services de la structure sont représentés. On note ainsi 28 personnes issues de différents services planning (en fonction de la division produit), 8 personnes des services logistiques et supply chain, 5 du contrôle production, 1 de la gestion des comptes, 11 de services d'encadrement et 29 services non renseignés. Les statuts professionnels mettent en exergue les réponses de 35 employés, 4 superviseurs, 27 managers, 4 Top Managers et12 réponses non fournies. Enfin, à la question du nombre d'années de carrière, elle est de 1 an minimum et 32 ans maximum au sein d'Alpha et de 2 ans minimum et 45 ans sur la carrière globale.

Concernant l'échelle appliquée à la liste des items proposés, elle se présente par une échelle de mesure en cinq points de type Likert de 'non important' à 'très important'. Cependant, une sixième colonne a été ajoutée. Elle est intitulée "non applicable" afin de donner la possibilité de " rejeter » une proposition pour une action. Le questionnaire comprend en tout 75 items.

La structure « originale » du questionnaire se présente telle que :

Thème 1 : Rôle du Top Management et de la politique SCM

Thème 2 : Le design du produit et services

Thème 3 : Implication des parties prenantes dans le SCM (hors clients finaux)

Thème 4 : Le SCM et les clients finaux

Thème 5 : Rôle de la cellule qui est en charge avec le déploiement du SCM

Thème 6 : Management des procédures - processus

Thème 7 : Qualité des données et des reportings

Thème 8 : Relations avec les employés

Thème 9 : Formation

Le nombre de réponses obtenues a permis d'utiliser deux types de traitement sous SPSS : un tri à plats et une ACP. La première étape, qui consiste à présenter les résultats sous forme de fréquences (par comptage des réponses), permet de réfléchir sur le profil des items et des thèmes phares à retenir. La seconde étape fait ressortir une hiérarchie des items au sein de chaque thème selon l'importance qui leur est accordée.

3 - Selon nous, nous devons considérer chaque employé comme un client.
Au sein de ce premier axe, nous suggérons d'intégrer deux thèmes :

- Un premier thème qui combine le thème «rôle du Top Management et de la politique $\mathrm{SCM}$ » et le thème « rôle de la cellule en charge du déploiement du SCM ». Cette combinaison part du constat de la place relativement éloignée $\left(6^{\text {ème }}\right.$ position : tri à plats) du thème 'rôle de la cellule en charge de déploiement du SCM' qui suggérerait de le rattacher au même thème que le 'rôle du Top Management dans la politique SCM'. Ce thème traiterait alors de 1 '“installation du SCM par le Top Management et le Département Dédié",

- Un deuxième thème : "qualité des données et des reportings" qui présente l'évolution $\mathrm{du}$ SCM. Ce thème considère qu'une information synthétique (au travers, par exemple, d'indicateurs clés) rend possible une réponse aux diverses requêtes de chaque client ${ }^{3}$ dans les délais les plus courts possibles. Sa place dans l'axe stratégique : mise en place et suivi tendrait à légitimer les métriques associées au SCM et qui - selon les résultats obtenus ( $7^{\text {ème }}$ position : tri à 
Tableau 4 : Tableau récapitulatif sur les résultats obtenus à travers le tri à plats et l'Analyse en Composantes Principales

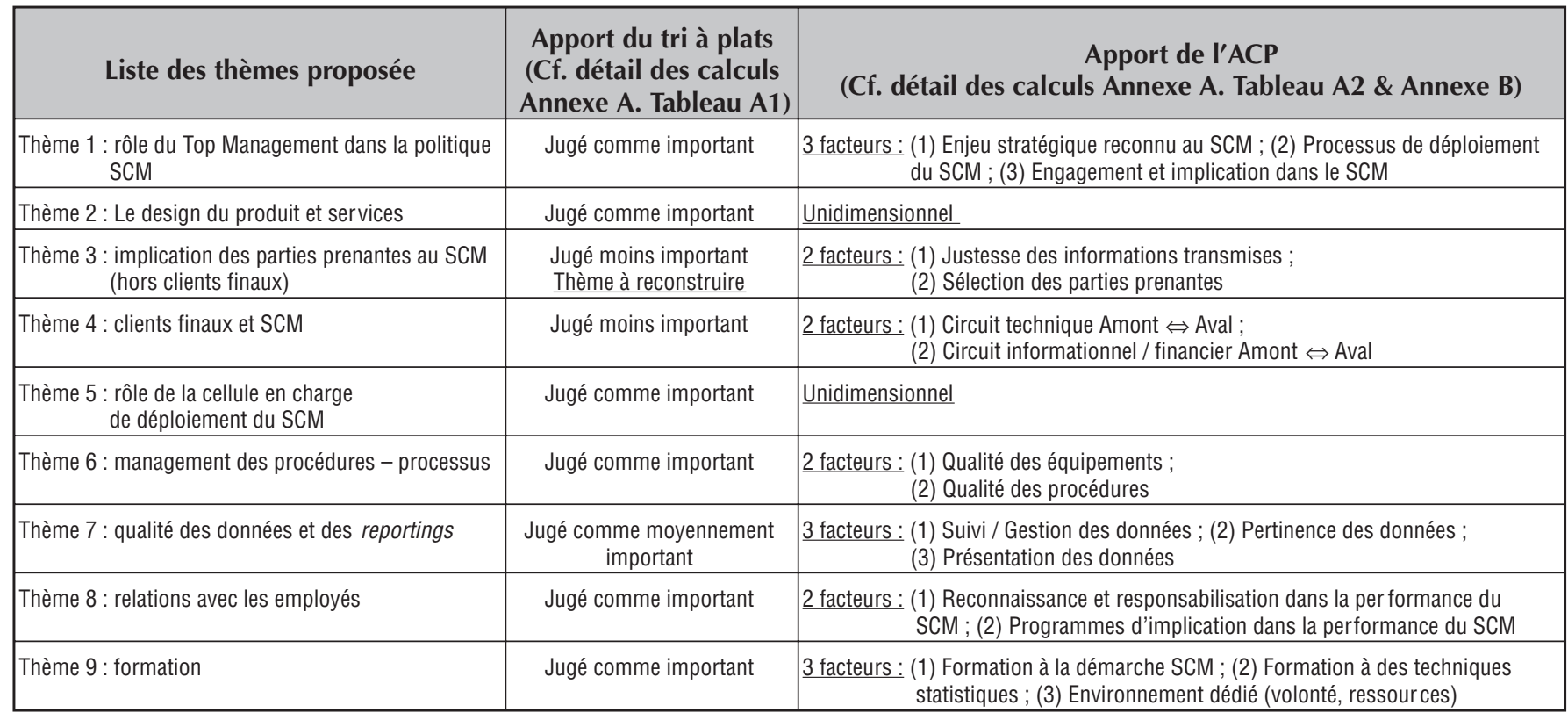

plats) - doivent faire l'objet d'une attention dans son mode de diffusion (qui diffuse ?, quoi diffuser?, comment calculer ?).

\section{2. l'Axe Socio-Organisationnel}

Pour assurer la mise en œuvre et la continuité du SCM, les employés doivent en comprendre ses objectifs. Ils doivent détenir des compétences transversales, aptes à les aider à articuler les différentes sphères d'activités du SCM. Donc, la philosophie du SCM ne doit pas uniquement toucher la traçabilité du produit, mais requiert de penser à toutes les interfaces transactionnelles induites par lui : compétences informatiques, financières, commerciales, en gestion de stocks, etc. Ce deuxième point trouve toute sa justification dans les idées clés telles que soulignées dans la rubrique « impacts humains au sens intra-organisationnel » du tableau 2.

$\mathrm{Au}$ sein de ce deuxième axe orienté socio-organisationnel, nous suggérons d'intégrer deux thèmes, à savoir le thème « formation » et le thème « relations avec les employés ».

\section{3. l'Axe Technologique}

La planification, l'ajustement et le suivi des flux de matières et d'informations associées sont les éléments de base d'une chaîne d'approvisionnement. Plus précisément, la gestion des processus logistiques impose des contrôles des flux de produits et de services associés, ceci afin de détenir un avantage compétitif sur le court et le long terme. Rappelons à ce titre que le thème 'Assemblage du produit et service fourni' est considéré comme unidimensionnel et donc souligne l'importance d'un contrôle complet du process productif. Dans un même sens, le contrôle de l'assemblage du produit passe nécessairement par une qualité des équipements et des procédures. Le résultat obtenu montre ainsi que le flux de produit s'établit principalement par une maintenance préventive des équipements $(67.6 \%$ de variance). Ce troisième point trouve toute sa justification dans les idées clés telles que soulignées dans la rubrique « impacts technologiques » $\mathrm{du}$ tableau 2.

Ce troisième axe orienté technologique comprendrait les thèmes «le design du produit et services » et « management des procédures processus ».

\section{4. l'Axe Capacité de réponse}

Concernant le dernier mais non le moindre axe !, une question se pose : pourquoi les deux thèmes qui le composent semblent-ils moins importants aux répondants ? En effet, pourquoi les individus considèrent-ils principalement ces thèmes comme 'moyennement important'. Selon nous, on peut y voir trois éléments. Premièrement, pour les employés, il est plus important de clarifier les processus et d'évaluer les compétences au sein de chaque firme, ceci avant d'envisager des liens avec des acteurs externes. Deuxièmement, il 
Figure 1 : Les axes de progrès du SCM

\begin{tabular}{|c|c|c|c|}
\hline & \multicolumn{2}{|c|}{ Axes de progrès du SCM } & 4 \\
\hline $\begin{array}{l}\text { Axe stratégique : } \\
\text { Mise en place } \\
\text { et suivi }\end{array}$ & $\begin{array}{l}\text { Axe socio- } \\
\text { organisationnel }\end{array}$ & $\begin{array}{l}\text { Axe } \\
\text { technologique: }\end{array}$ & $\begin{array}{l}\text { Axe capacité de } \\
\text { réponse : }\end{array}$ \\
\hline 1) Rôle du Top & $\begin{array}{l}\text { 1) Formation } \\
\text { 2) Relations avec }\end{array}$ & $\begin{array}{l}\text { 1) Produit et service } \\
\text { fournis }\end{array}$ & $\begin{array}{l}\text { 1) Clients finaux } \\
\text { 2) Autres parties }\end{array}$ \\
\hline Département dédié & les empioyes & $\begin{array}{l}\text { 2) Gestion des } \\
\text { processus - }\end{array}$ & prenantes \\
\hline $\begin{array}{l}\text { 2) Qualité des } \\
\text { données et } \\
\text { reportings }\end{array}$ & & procédures & \\
\hline
\end{tabular}

peut être considéré que la satisfaction des acteurs externes (tout particulièrement le client final) passe initialement par une coordination des départements internes à la firme. Dernièrement, et en s'appuyant sur certains commentaires, la notion de parties prenantes peut être très large et, de fait, est-il difficile de 'personnaliser' une réponse pour un groupe d'acteurs trop conséquent. Ce quatrième point trouve toute sa justification dans les idées clés telles que soulignées dans la rubrique « impacts humains au sens inter-organisationnel » du tableau 2.

Ce quatrième axe dont l'orientation se situerait sur la capacité de réponse comprendrait le thème relatif aux « clients finaux » et celui relatif aux « autres parties prenantes », remarquant que pour ce dernier thème, une reformulation voire une refonte totale serait nécessaire.

A cet égard, nous proposons le schéma 'final' (Cf. figure 1).

\section{Conclusion}

Dans ce papier, nous avons identifié plusieurs approches pour décrire les dimensions du Supply Chain Management (SCM). De cette analyse, nous avons pu extraire une matrice mettant en exergue des éléments fondamentaux à une analyse quantitative. Dans notre réflexion, nous avons utilisé comme base de notre étude un questionnaire testé empiriquement auprès de 292 entreprises (Forker et al., 1997). Celui-ci décrit la performance du Total Quality Management dans un contexte de chaîne d'approvisionnement. Après plusieurs modifications, telles que décrites dans notre méthodologie, afin de le rendre plus 'proche' d'une approche SCM, nous pensons que ce document fournit des données utiles au Top Management de chaque firme qui adopte un
SCM, de même qu' une base de réflexion pour les chercheurs en gestion.

Selon nous, cette étude a des implications tant sur le plan académique que managérial. Pour les gestionnaires, le questionnaire permet une conceptualisation par thème du SCM, mais surtout d'avoir connaissance des perceptions d'employés d'une organisation multinationale - pivot de sa chaîne d'approvisionnement. Il devrait faciliter la mise en œuvre du SCM au sein et hors de la firme. La classification de chaque thème par importance et l'analyse factorielle sont des supports pertinents pour la concrétisation du SCM. Avec notre architecture, les chercheurs en gestion peuvent conceptualiser plus facilement les domaines du SCM. Sur ce point, les commentaires par quelques interviewés donnent des informations complémentaires intéressantes. Par exemple, la notion de 'parties prenantes' n'est pas suffisamment détaillée. En effet, pour chaque personne, la signification peut être différente : cela peut être une autre fonction, une filiale ou un distributeur, etc. Une définition claire du terme 'partie prenante' au sein de chaque item apparaît comme nécessaire (sur ce point, il serait possible de prendre la 'classification' donnée par la définition du CSCMP donnée dans l'introduction : “....fournisseurs, intermédiaires, prestataires de service et clients ....". En outre, et de manière plus générale, il pourrait être intéressant d'inclure un ou plusieurs items sur la logistique des retours.

\section{Bibliographie}

Bowersox, D., Closs, D. et Stank, T. (1999), $21^{\text {st }}$ Century Logistics: Making Supply Chain Integration a Reality, Council of Logistics Management, Oak Brook (IL).

Cooper, M., Lambert, D. et Pagh, J. (1997), Supply Chain Management: more than a New Name for Logistics, The International Journal of Logistics Management, Vol. 8, $\mathrm{n}^{\circ} 1$, pp. 1-13.

Forker, L., Mendez, D. et Hershauer, J. (1997), Total Quality Management in the Supply Chain: What is its Impact on Performance?, International Journal of Production Research, Vol. 35, n 6, pp. 1681-1701.

Flynn, B. et Flynn, E. (2005), Synergies between Supply Chain Management and Quality Management: Emerging Implications, International Journal of Production Research, Vol. 43, n 16 , pp. 3421-3436. 
Gibson, B., Mentzer, J. et Cook, R., (2005), Supply Chain Management: the Pursuit of a consensus definition, Journal of Business logistics, Vol. 26, $\mathrm{n}^{\circ} 2$, pp. 17-25.

GRLT - Global Logistics Research Team (1995), World Class Logistics: the challenge of managing continuous change, Council of Logistics Management, Oak Brook (IL).

Lambert, D., Cooper, M. et Pagh, J. (1998), Supply Chain Management: Implementation Issues and Research Opportunities, The International Journal of Logistics Management, Vol. 9, $\mathrm{n}^{\circ}$ 2, pp. 1-19.
Mentzer, J., DeWitt, W., Keebler, J., Min, S., Nix, N., Smith, C. et Zacharia, Z. (2001), Defining Supply Chain Management, Journal of Business Logistics, Vol. 22, n 2, pp. 1-25.

Min, S. et Mentzer J. (2004), Developing and Measuring Supply Chain Management Concepts, Journal of Business Logistics, Vol. 25, $\mathrm{n}^{\circ} 1$, pp. 63-91.

Wagoner, S. (1989), Logistics and Quality Management, Logistics Spectrum, Vol. 23, $\mathrm{n}^{\circ} 4$, pp. 13-16.

\section{Annexe A}

Tableau A1 : La classification par fréquence de chaque thème

\begin{tabular}{|c|c|c|c|c|c|c|c|c|c|c|c|c|c|}
\hline Liste des thèmes proposée & Nb It. & NA & MV & $1+2$ & $\%$ & 3 & $\%$ & 4 & $\%$ & Rang & 5 & $\%$ & Rang \\
\hline $\begin{array}{l}\text { Thème 1: rôle du Top Management dans la } \\
\text { politique SCM }\end{array}$ & 13 & 32 & 6 & 70 & 5.4 & 210 & 16.2 & 299 & 23 & & 499 & 34.5 & 1 \\
\hline Thème 2: le design du produit et services & 6 & 17 & 2 & 77 & 12.8 & 116 & 19.3 & 125 & 20.8 & & 155 & 25.8 & 3 \\
\hline $\begin{array}{l}\text { Thème 3: implication des parties prenantes au } \\
\text { SCM (hors clients finaux) }\end{array}$ & 8 & 48 & 6 & 109 & 13.6 & 214 & 26.8 & 168 & 21 & & 111 & 13.9 & 9 \\
\hline Thème 4: clients finaux et SCM & 5 & 13 & 5 & 49 & 9.8 & 94 & 18.8 & 135 & 27 & & 114 & 22.8 & 8 \\
\hline $\begin{array}{l}\text { Thème 5: rôle de la cellule en charge de } \\
\text { déploiement du SCM }\end{array}$ & 5 & 18 & 2 & 32 & 6.4 & 99 & 19.8 & 144 & 28.8 & & 115 & 23 & 6 \\
\hline $\begin{array}{l}\text { Thème 6: management des procédures - } \\
\text { processus }\end{array}$ & 6 & 24 & 2 & 50 & 8.3 & 109 & 18.2 & 160 & 26.7 & 5 & 147 & 24.5 & 4 \\
\hline $\begin{array}{l}\text { Thème 7: qualité des données et des } \\
\text { reportings }\end{array}$ & 16 & 43 & 9 & 133 & 8.3 & 336 & 21 & 424 & 26.5 & & 367 & 22.9 & 7 \\
\hline Thème 8: relations avec les employés & 8 & 2 & 1 & 103 & 12.9 & 108 & 13.5 & 246 & 30.8 & 4 & 196 & 24.5 & $4^{\prime} \Rightarrow>5$ \\
\hline Thème 9: formation & 8 & 4 & 2 & 96 & 12 & 140 & 17.5 & 202 & 25.3 & & 212 & 26.5 & 2 \\
\hline
\end{tabular}

Légende: MV: valeur manquante; N.A.: non applicable; 1: non important ; 2: peu important ; 3: moyennement important ; 4: assez important ; 5: très important

\section{Tableau A2 : La classification par l'analyse factorielle}

\begin{tabular}{|c|c|c|c|}
\hline Liste des thèmes présentée & Nombre de facteurs & Variance & $\begin{array}{c}\alpha \text { de } \\
\text { Cronbach }\end{array}$ \\
\hline Thème 1: Rôle du top management dans la politique SCM & $\begin{array}{l}\text { - Enjeu stratégique reconnu au SCM } \\
\text { - Processus de déploiement du SCM } \\
\text { - Engagement et implication dans le SCM } \\
\end{array}$ & $\begin{array}{c}54.2 \% \\
10.2 \% \\
7.0 \% \\
\end{array}$ & $\begin{array}{l}.8895 \\
.8360 \\
.8536 \\
\end{array}$ \\
\hline Thème 2: Le design du produit et services & Unidimensionnel & & .8379 \\
\hline $\begin{array}{l}\text { Thème 3: Implication des parties prenantes au SCM } \\
\text { (hors clients finaux) }\end{array}$ & $\begin{array}{l}\text { - Justesse des informations transmises } \\
\text { - Sélection des parties prenantes }\end{array}$ & $\begin{array}{l}57.6 \% \\
12.0 \% \\
\end{array}$ & $\begin{array}{l}.8648 \\
.8234\end{array}$ \\
\hline Thème 4: Les clients finaux et le SCM & $\begin{array}{l}\text { - Circuit technique Amont ó Aval } \\
\text { - Circuit informationnel / financier Amont ó Aval }\end{array}$ & $\begin{array}{l}55.0 \% \\
17.3 \% \\
\end{array}$ & $\begin{array}{l}.7887 \\
.6594\end{array}$ \\
\hline Thème 5: Rôle de la cellule en charge du déploiement du SCM & Unidimensionnel & & .9037 \\
\hline Thème 6: Management des processus - procédures & $\begin{array}{l}\text { - Qualité des équipements } \\
\text { - Qualité des procédures }\end{array}$ & $\begin{array}{l}67.6 \% \\
10.8 \% \\
\end{array}$ & $\begin{array}{l}.8672 \\
.8468\end{array}$ \\
\hline Thème 7: Qualité des données et reportings & $\begin{array}{l}\text { - Suivi / Gestion des données } \\
\text { - Pertinence des données } \\
\text { - Présentation des données }\end{array}$ & $\begin{array}{c}55.5 \% \\
10.3 \% \\
7.2 \% \\
\end{array}$ & $\begin{array}{l}.9160 \\
.9316 \\
.8750 \\
\end{array}$ \\
\hline Thème 8: Relations avec les employés & $\begin{array}{l}\text { - Reconnaissance et responsabilisation dans la per formance du SCM } \\
\text { - Programmes d'implication dans la performance du SCM }\end{array}$ & $\begin{array}{l}71.8 \% \\
7.8 \% \\
\end{array}$ & $\begin{array}{l}.9273 \\
.8802 \\
\end{array}$ \\
\hline Thème 9: Formation & $\begin{array}{l}\text { - Formation à la démarche SCM } \\
\text { - Formation à des techniques statistiques } \\
\text { - Environnement dédié (volonté, ressources) }\end{array}$ & $\begin{array}{l}63.6 \% \\
3.2 \% \\
7.7 \%\end{array}$ & $\begin{array}{l}.9307 \\
.8308 \\
.8223\end{array}$ \\
\hline
\end{tabular}




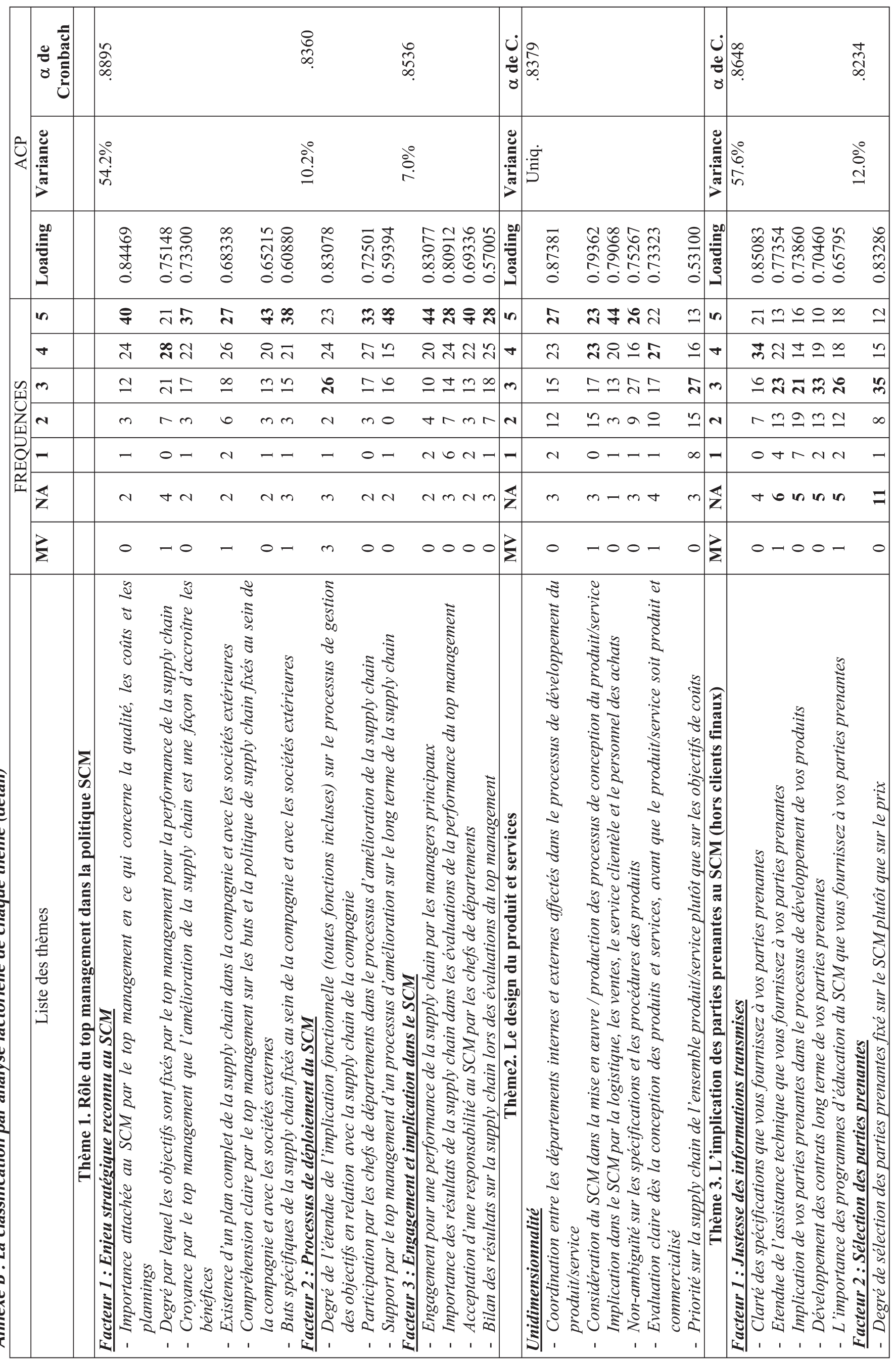




\begin{tabular}{|c|c|c|c|c|c|c|c|c|c|c|c|c|c|}
\hline & 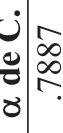 & ठี & & 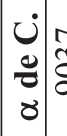 & & 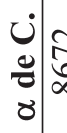 & $\begin{array}{l}1 \\
\frac{1}{0} \\
\infty \\
\infty\end{array}$ & $\begin{array}{l}\infty \\
\infty \\
\infty \\
\infty \\
\infty\end{array}$ & $\begin{array}{l}ن \\
0 \\
0 \\
0\end{array} \mid$ & & $\frac{\sigma}{\sigma}$ & $\frac{\substack{n \\
\infty}}{\infty}$ & \\
\hline & 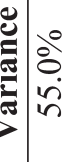 & $\stackrel{\text { ले }}{\stackrel{2}{s}}$ & & 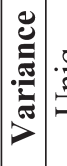 & & 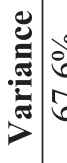 & $\begin{array}{l}\dot{0}_{0}^{\circ} \\
\text { b }\end{array}$ & $\stackrel{\varrho}{0}$ & 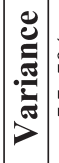 & & $\stackrel{\circ}{\stackrel{0}{े}}$ & $\stackrel{\stackrel{े}{~}}{r}$ & \\
\hline 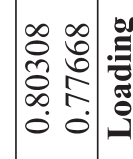 & |م & 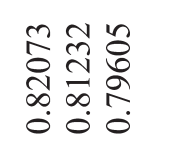 & 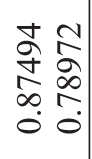 & 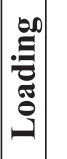 & 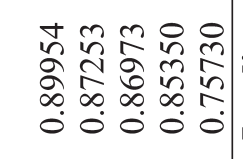 & :00 & 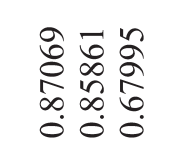 & 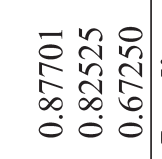 & 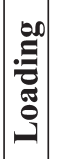 & 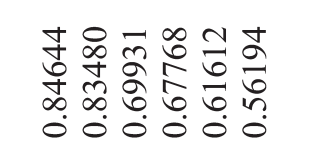 & 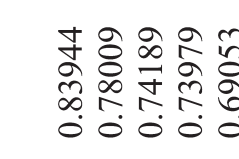 & & 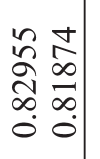 \\
\hline $\pm n$ in & $n$ & 녹 & $\bar{m} \simeq$ & in & $\because$ உக் & is & $\widetilde{N} \cong \infty$ & సి ర స & in & $\bar{\sim} \stackrel{\sim}{0} \underset{\sim}{\sim} \stackrel{\sim}{\sim}$ & "ెస్లిల్ & & $\stackrel{\infty}{\sim}$ \\
\hline ปั งิ| & + & $\ddot{\sim} \mathscr{\sim}$ & خે & $\nabla$ & 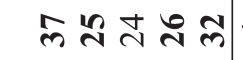 & $\nabla$ & মัล & $\hat{\sim} \infty$ & $\nabla$ & సిনిળల్లి స̃ & ટ્స థ્సి ల్లి స & & กิ ผ \\
\hline ते लm & $m$ & $\stackrel{\infty}{\sim} \mathscr{\sim}$ & $\because \pm$ & $\infty$ & $\vec{\sim} \frown \pm \vec{\sim}$ & $\infty$ & สํㅡ & $\simeq \because$ & $\infty$ & $\vec{\sim} こ \mathscr{N} ニ 尺 ゚$ & ニぇュュ & & సิ \\
\hline$\sigma \cong N$ & v & $-\infty a$ & $\nabla \pm$ & $\sim$ & $n$ in $n$ in $m$ & $N$ & $-\infty \infty$ & $\circ 0+$ & $\mathbf{N}$ & $+\infty \subseteq \infty \subseteq 0$ & $\forall n \circ \infty n$ & & or \\
\hline$-n-$ & -1 & $N m-$ & -0 & - & $-N-m-$ & - & -00 & $-m N$ & - & $\forall m+\forall N N$ & $-T N-$ & & $m+$ \\
\hline ○ $6 \mid \frac{\pi}{Z}$ & $\mathbb{z}$ & $m+n$ & $-m$ & $\mathbb{z}$ & $m+\forall m+$ & $\mathbb{z}$ & $\ln$ int & $m m t$ & $\overleftrightarrow{z}$ & $m m m m+N$ & $m \sim N N O$ & & \\
\hline$N \sim \vec{z}$ & $\sum$ & --- & -- & $\vec{z}$ & $0-1-00$ & 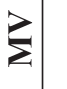 & -00 & $00-1$ & $\mathbf{z}$ & OOTnOO & OOMno & & \\
\hline 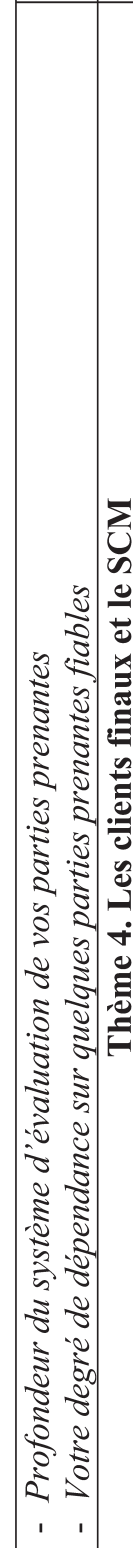 & 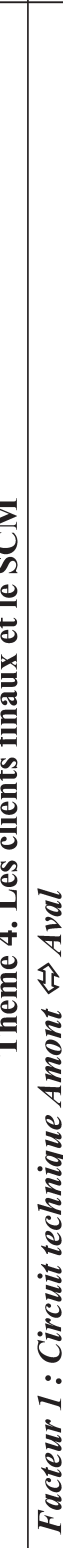 & 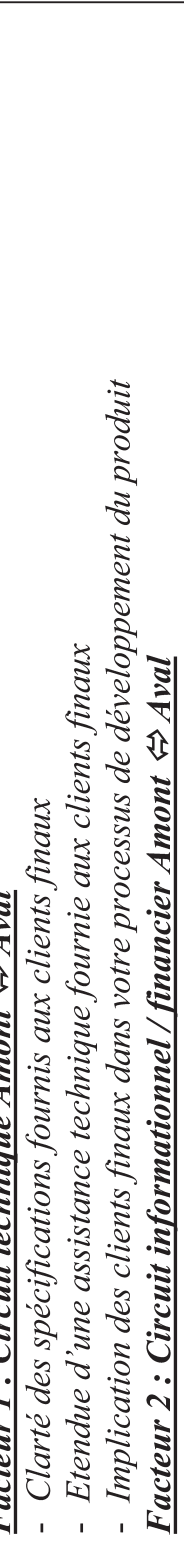 & 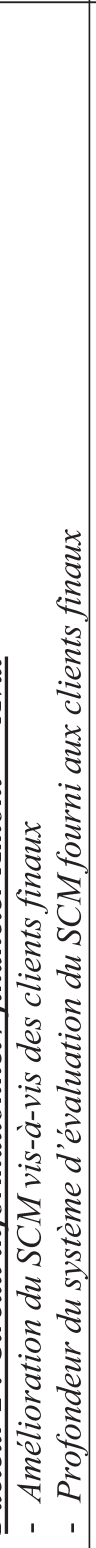 & 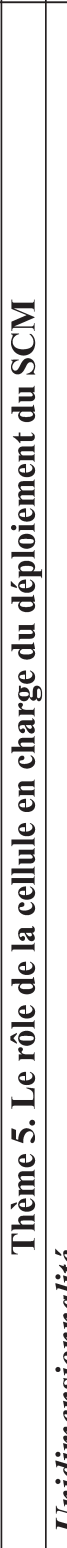 & 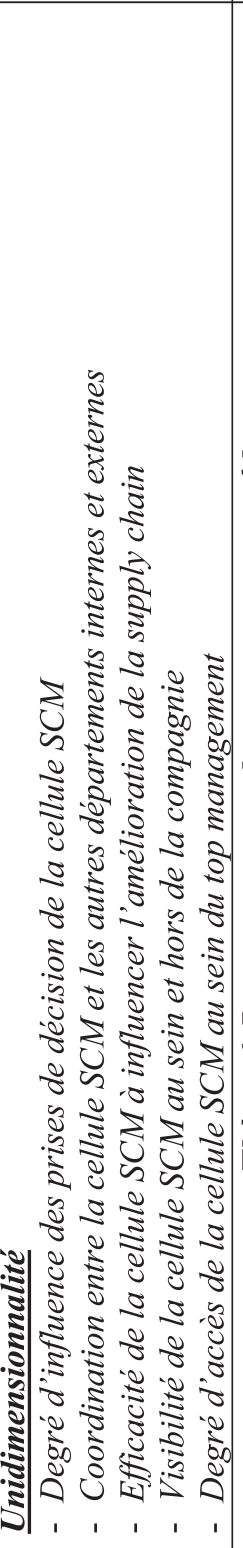 & 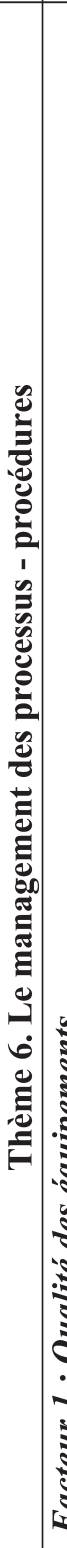 & 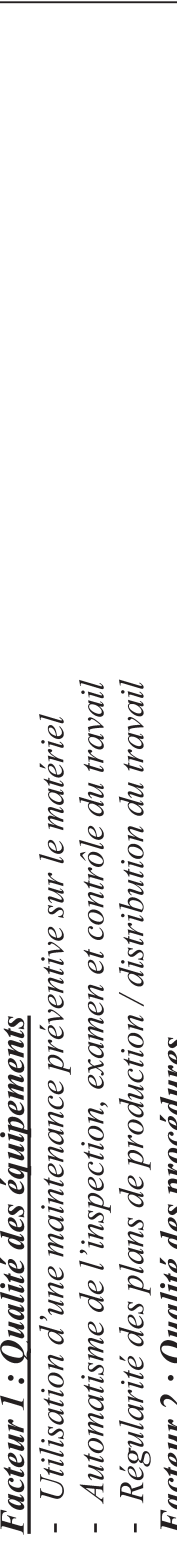 & 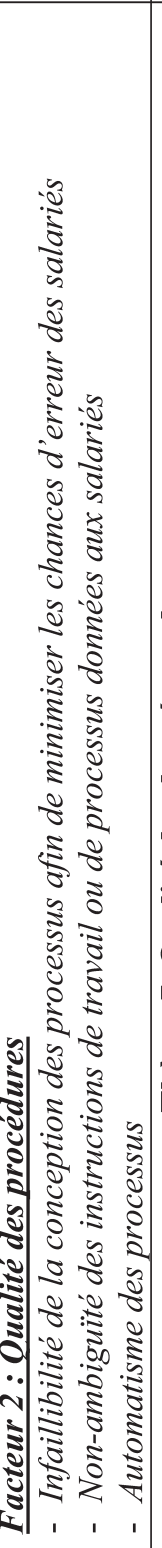 & 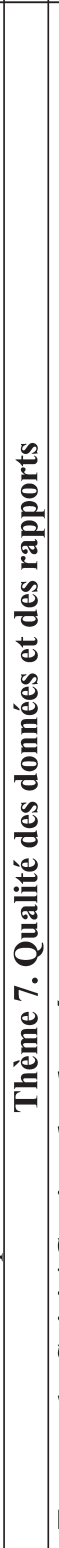 & 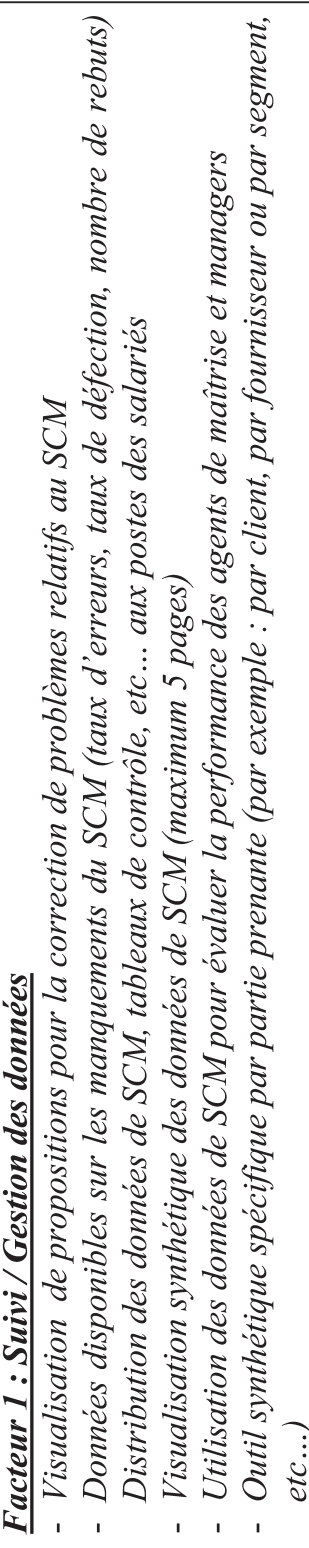 & 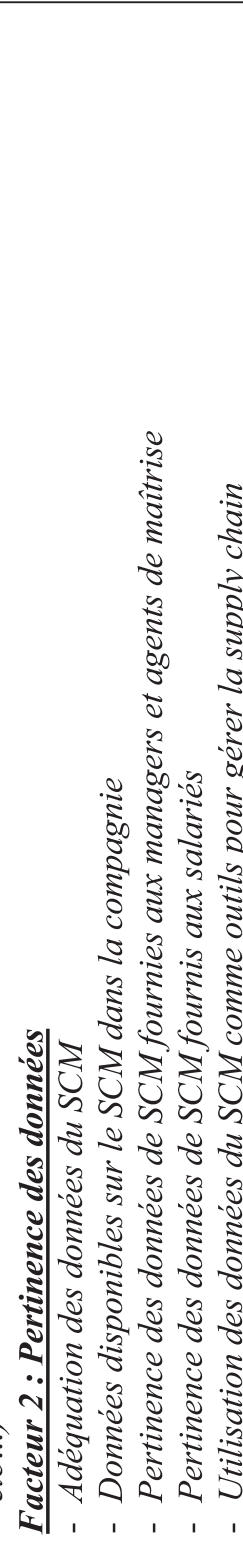 & 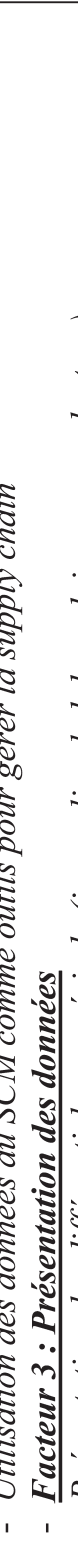 & 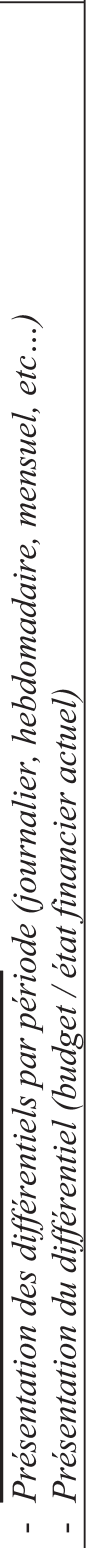 \\
\hline
\end{tabular}




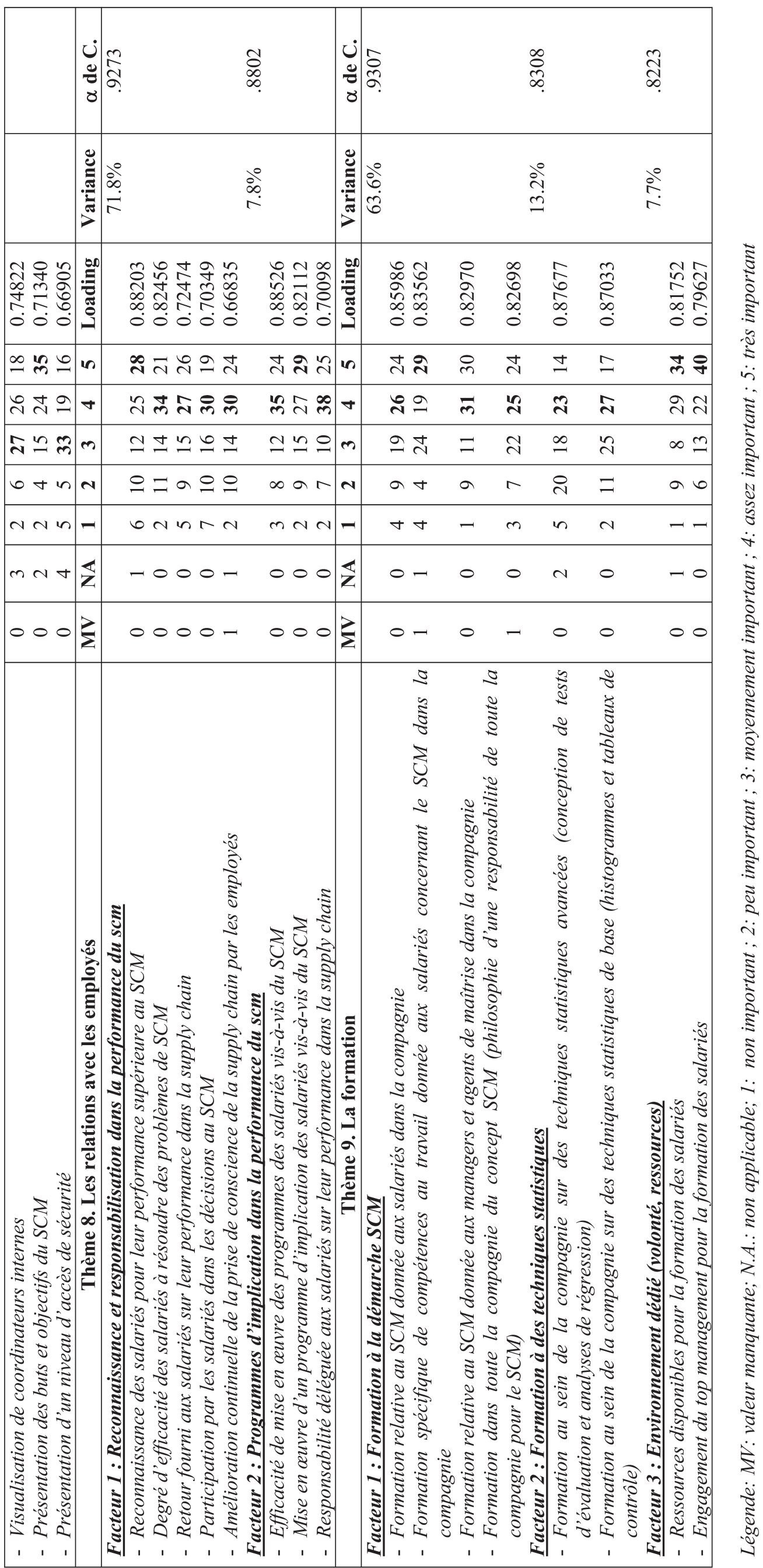

